Gut, 1988, 29, 686-688

Case report

\title{
Devastating diarrhoea caused by azathioprine: management difficulty in inflammatory bowel disease
}

\author{
J COX, T K DANESHMEND, C J HAWKEY, R F A LOGAN, \\ AND R P WALT \\ From the Department of Therapeutics, University Hospital Nottingham, Nottingham.
}

SUMMARY Azathioprine toxicity complicated the management of four patients with inflammatory bowel disease. All patients received the drug as adjunctive therapy to steroids when responses to the latter were poor. After a variable sensitising period the patients developed severe diarrhoea and abdominal pain and this was believed at first to be a manifestation of their underlying diseases but rechallenge with azathioprine reproduced the problem. During three episodes described emergency admission to hospital and resuscitation with intravenous fluids was required. The cases illustrate the difficulty clinicians have in recognising drug induced effects which mimic the underlying disease. When a patient suspects a reaction to azathioprine we believe any rechallenge should only be undertaken in the controlled hospital environment.

Azathioprine is an immunosuppressive drug which is metabolised to 6 mercaptopurine and after transformation to the ribonucleotide it interferes with all stages of purine metabolism. Dose related side effects, particularly bone marrow suppression are well recognised. Idiosyncratic reactions have been described, notably an acute shock syndrome. We have used azathioprine as additional treatment for patients with inflammatory bowel disease relatively unresponsive to conventional medication. In the four cases reported here, acute hypersensitivity to azathioprine caused symptoms which were difficult to differentiate from an exacerbation of the underlying inflammatory bowel disease and in at least one case led to inappropriate management.

\section{Case reports}

PATIENT 1

A 31 year old man with ulcerative colitis diagnosed by barium enema, sigmoidoscopy and biopsy in June 1986 was initially treated with colifoam enemata and then oral prednisolone, plus mesalazine (he was intolerant of sulphasalazine). Azathioprine was

Address for correspondence: R P Walt. Department of Medicine, Queen Elizabeth Hospital, Birmingham B15.

Received for publication 2 November 1987 added (October $1986100 \mathrm{mg}$ daily) as it was proving difficult to reduce his steroid dose below $20 \mathrm{mg}$ daily without worsening symptoms. Eventually symptoms settled on this regimen and he continued to take azathioprine and mesalazine until January 1987 when he spontaneously stopped all medication. Three weeks later his diarrhoea had returned and prednisolone and mesalazine were reintroduced. After two weeks, however, there had been only a poor response and azathioprine was restarted. Two hours after the first dose of $50 \mathrm{mg}$ he experienced severe colicky abdominal pain with nausea, vomiting, and rigors followed by profuse bloody diarrhoea, passing 20 motions in 24 hours. Sixteen hours later he was admitted to hospital with an unrecordable blood pressure and severe dehydration. He was apyrexial with a normal haemoglobin, white count, and ESR of 11. Repeated blood, stool and urine cultures were negative. Serum amylase was normal. Sigmoidoscopy revealed a severely inflamed rectum. The hypotension responded to intravenous fluids, and bowel habit settled over the next two days to the previous level of 10 bowel motions a day. Despite intravenous steroids (64 $\mathrm{mg}$ prednisolone daily), oral mesalazine and an elemental diet his condition failed to improve. The patient was reluctant to undergo surgery and azathioprine was restarted in a lower 
dose. Ninety minutes after a $25 \mathrm{mg}$ dose he developed symptoms identical to those which prompted his admission to hospital with profuse bloody diarrhoea (5 litres in 24 hours), and hypotension (systolic 60 $\mathrm{mmHg}$ ). He was resuscitated but during the following two weeks his condition deteriorated despite continued high dose steroids and parenteral nutrition. He agreed to colectomy which showed severe active total colitis. Postoperative recovery was uneventful.

\section{PATIENT 2}

A 73 year old woman had a two year history of total ulcerative colitis diagnosed after sigmoidoscopy, barium enema and rectal biopsy. In order to control symptoms continual prednisolone was required and she was troubled by Cushingoid side effects and heart failure. Azathioprine (100 mg/day) was introduced in December 1986 in an attempt to reduce steroid dosage. She stopped the azathioprine after two weeks, however, because of 'sickness'. In March 1987 when taking prednisolone $10 \mathrm{mg}$ daily, mesalazine and Frumil she was admitted to hospital with an exacerbation of colitis. During this admission another attempt was made to reduce the steroid dose by reintroducing azathioprine $(25 \mathrm{mg})$. Two hours after taking the first dose she complained of severe abdominal pain associated with nausea and vomiting and followed by a worsening of her diarrhoea. No further doses were given and she returned to her previous level of symptoms in 24 hours. The exacerbation gradually settled but she has continued to need prednisolone (10 $\mathrm{mg}$ or above).

\section{PATIENT 3}

This patient presented in 1981 aged 14 with rectal bleeding. Sigmoidoscopy and biopsy confirmed proctitis and barium enema at that time was normal. Two years later a barium enema showed disease to the splenic flexure. She had repeated exacerbations between 1983 and 1984 and oral prednisolone was started in October 1984. Two months later her condition was unchanged and azathioprine was added increasing over one week from $50 \mathrm{mg}-100 \mathrm{mg}$ daily. Four weeks after starting azathioprine she was admitted to hospital as an emergency with watery, bloody diarrhoea (hourly during the day and three times at night), abdominal pain and nausea. She was dehydrated and had postural hypotension (110/80 lying, 90/60 standing). Her treatment regimen then was prednisolone $20 \mathrm{mg}$ daily, azathioprine $100 \mathrm{mg}$ daily and sulphasalazine $4 \mathrm{G}$ daily. Intravenous prednisolone and fluids were started, all oral medication including azathioprine was stopped and within four days stool frequency had reduced to once daily. Oral prednisolone was tailed off uneventfully over three weeks. No infective cause was detected but the clinical diagnosis was thought to have been infective diarrhoea. Over the next two years she suffered several mild exacerbations which settled quickly on local steroids. In December 1986, an exacerbation started and prednisolone was given orally. Relapses occurred whenever the dose was reduced below 10 mg and in July 1987 she was admitted for intensive therapy. Azathioprine was also given $(50 \mathrm{mg}$ daily increasing to $150 \mathrm{mg}$ daily) and an initial improvement was noted. One week later, however, stool frequency of watery blood stained motions increased to $10 / 24$ hours and she developed generalised colicky abdominal pain, nausea, and a fever $\left(39^{\circ} \mathrm{C}\right)$. Sigmoidoscopy confirmed active proctitis and abdominal radiographs showed faecal retention on the right side of the colon with an otherwise normal gaseous pattern. Searches for infective causes proved negative. Azathioprine was stopped and four days later bowel frequency was once daily. Prednisolone therapy was decreased over the next three weeks without difficulty.

\section{PATIENT 4}

A 30 year old man was diagnosed as having Crohn's disease of the terminal ileum associated with a moderate terminal ileal stricture in July 1986 . Over the following nine months he had recurrent episodes of abdominal discomfort which responded to short courses of prednisolone but recurred shortly after each course. The frequency and severity of his symptoms were not considered bad enough to warrant surgery. In March 1987 azathioprine $(50 \mathrm{mg}$ tds) was introduced to try and improve the control of his symptoms. Two weeks later he was admitted to a surgical ward with severe generalised abdominal pain associated with nausea and vomiting, and diarrhoea which was not a previous feature of his condition. He was ill enough to require intravenous fluids but haematological indices suggested the Crohn's disease was not active and the serum amylase was normal. Blood cultures were negative. Azathioprine was discontinued and all symptoms settled. Steroid dosage was reduced rapidly and he has remained well off all medication.

\section{Discussion}

The role of azathioprine in the treatment of difficult ulcerative colitis is unclear. In the most recent published study Kirk and Lennard-Jones reported a steroid sparing effect in patients requiring continuous treatment with oral steroids. ${ }^{1}$ A number of dose dependent side effects, such as leucopenia and nausea, are well recognised, and reversible on stopping the drug. ${ }^{2}$ Such effects result in about $10 \%$ of 
patients having treatment withdrawn. Nevertheless, as an immunosuppressant, azathioprine is reputed to be one of the safest drugs of its type in widespread use. ${ }^{3}$ During the two decades to 1984 only 328 reports of suspected reactions had been received worldwide by the manufacturers. ${ }^{3}$

Azathioprine is a recognised cause of acute pancreatitis ${ }^{4}$ and a shock like syndrome with a wide spectrum of symptoms including: fever, rash, nausea, general malaise, rigors, and hypotension. ${ }^{56}$ The first patient illustrates this acute life threatening hypersensitivity reaction which is independent of dose. It has occurred with doses as low as $12.5 \mathrm{mg}$. ${ }^{7}$ Although diarrhoea has previously been mentioned, its importance has not been emphasised. In one report diarrhoea, nausea, and abdominal pain started four weeks after introduction of azathioprine in a patient with quiescent colitis and recurred on rechallenge. ${ }^{8}$ It has been pointed out that pancreatitis has predominantly been recorded in patients with inflammatory bowel disease whereas the hypersensitivity/shock syndrome has generally been noted in patients with rheumatoid arthritis or systemic lupus. ${ }^{3}$ This may suggest, at first glance, that different hazards of the drug become manifest in different clinical circumstances but it is more likely that the discrepancy of reporting reflects clinicians interests and awareness of side effects.

We believe that our cases represent true drug toxicity but proof of this is virtually impossible to gather. This difficulty arises because the symptoms of toxicity mimic closely those expected with the disease. Indeed our clinical awareness of the problem was raised by the dramatic effects of unwitting rechallenge in patient 1 . The other cases have since come to light and the recognition of toxicity was retrospective in two cases. Symptoms were ascribed to the underlying inflammatory bowel disease. In patients 1 and 3, rechallenge with azathioprine was inappropriate and indeed led to life threatening consequences. In all our patients the reaction occurred after an initial sensitising period of at least two weeks. It is interesting that the first patient had not previously complained of adverse effects during his initial three month course. The mechanism of toxicity is unclear. Hypersensitivity may be the explanation but delay in symptom onset as seen in case 4 and other reported cases suggest that this is not a wholly satisfactory explanation. Diarrhoea of these proportions is not generally a feature of other drug hypersensitivity reactions. It is possible that only patients with underlying bowel disease display the symptom, but the possibility remains that in some patients the drug stimulates intestinal secretion.

These cases show the problem of differentiating between an exacerbation of the underlying disease process, an infective diarrhoea and a drug reaction. In three of the cases the patients reported their suspicion that the drug was the cause of their symptoms. As diarrhoea and malaise were usual complaints in their conditions, limited notice was taken of their suspicions. We believe that if diarrhoea worsens after starting therapy azathioprine should be stopped as it may be aggravating the problem. There also seems little point in continuing its use after three months if clinical response and steroid sparing are negligible. If patients suspect a reaction they should be taken seriously and rechallenge in hospital should only be undertaken if an absolute clinical indication for azathioprine use exists. Previous uncomplicated use of the drug in any individual does not exclude the possibility of subsequent serious toxicity.

\section{References}

1 Kirk AP, Lennard-Jones JE. Controlled trial of azathioprine in chronic ulcerative colitis. Br Med J 1982; 284: 1291-2.

2 Stenfert Kroese WF. Immunosuppressive drugs. In: Meyler L, Herxheimer A, eds. Meyler's side effects of drugs. Vol 7. Amsterdam: Excerpta Medica, 1972; 643.

3 Lawson DH, Lovatt GE, Gurton CS, Hennings RC. Adverse effects of azathioprine. Adverse Drug React Acute Poisoning Rev 1984; 3: 161-71.

4 Davis M, Eddleston A, Williams R. Hypersensitivity and jaundice due to azathioprine. Postgrad Med J 1980; 56: 274-5.

5 King J, Laver MC, Fairley KF, Ames GA. Sensitivity to azathioprine. Med J Aust 1972; 2: 939-41.

6 Cunningham T, Barraclough D, Muirden K. Azathioprine induced shock. $B r$ Med $J$ 1981; 283: 823-4.

7 Zaltman M, Kallenbach J, Shapiro T, et al. Life threatening hypotension associated with azathioprine therapy. $S$ Afr Med J 1984; 65: 306.

8 Jewell DP, Truelove SC. Azathioprine in ulcerative colitis: final report on controlled therapeutic trial. Br Med J 1974; iv: 627-30. 